\title{
Synonymous Keyword Auction Mechanism for Advertising Sites
}

\author{
Zhiwei Zhou, Junwu Zhu* \\ College of Information Engineering, Yangzhou University, Yangzhou 225127, China \\ * Corresponding Author: jwzhu@yzu.edu.cn
}

\begin{abstract}
The existing keywords auction mechanism with only a single keyword as the auction object, ignoring Web pages associated with synonyms, leads to low advertising recall rate and low profits of the search engine. Synonymous Keyword Auction Mechanism for Advertising Sites (SKMS) is proposed in this paper dealing with the above issues to realize allocation and payment of keyword and synonym set. Advertising sites allocation algorithm based on 0-1 matrix, which greatly extends an available set of auction object, takes account of synonymous keywords with search function and application value. Payment algorithm based on second-price guarantees truthfulness of the mechanism. Compared to existing keywords auction mechanism, SKMS not only improves recall rate, but also increases profit of search engine.
\end{abstract}

Keywords: advertisers, search engine, profit, synonymous keyword, advertising sites.

\section{Introduction}

With the rapid development of Internet, search engine has become an important tool to collect information quickly and accurately. More and more Internet users use search engines to search for information so that search engines provide keyword advertising services to improve the search engine's profit. Every time the search engine advertising auction system represented by Google has a value of hundreds of millions of advertising sites sold and millions of advertisers participate in the auction. The keyword auctions of novelty, and high profitability, the beginning has become a popular research topic in the world ${ }^{[1]}$.

On the current domestic and international development process of keyword auction, the first use is Generalized First-Price (GFP) ${ }^{[2]}$. The drawback of this auction mechanism is that any keyword is an independent auction entity, and advertisers can adjust the price at any time to deal with the reaction of competitors to cause greater price volatility. That is, the first price auction mechanism does not have a broad sense of authenticity. Then Google introduced Generalized Second-Price (GSP) ${ }^{[3]}$. The main difference between GFP and GSP is the payment method, which can solve the problem of the advertiser's price fluctuation. But GSP focus on single keywords auction problem and the allocation of advertising sites is based on the price of the distribution. This leads to demand dissatisfaction of advertisers, low recall of advertising and low gain of search engines.

In this paper, we will solve the problem of the allocation and payment of the advertising by Synonymy Keyword Auction Mechanism for Advertising (SKMS). SKMS guarantees computational efficiency, individual rationality and authenticity. SKMS allocation algorithm begins the auction with the keyword of the highest frequency (keyword of the highest frequency). The payment scheme of SKMS considers whether the situation that degree of advertising site is not less than 2 exists. If existing, the winning advertiser $v_{i}$ needs to pay the bid $b_{v_{i}^{\prime}}^{(j)}$ of an advertiser $v_{i}^{\prime}$ only next to the former. Otherwise, the winner needs to pay the value of keyword.

\section{Contribution to this paper:}

(1) In this paper, we consider the advertising auction of synonymous keywords so as to increase the opportunity for advertisers to get advertising and revenue from search engine advertising.

(2) SKMS is proved of truthfulness. $r\left(f^{(j)}\right)=$ $\left|L^{(j)}\right| \Leftrightarrow$ Advertising sites are completely allocated for keyword $u_{j}$.

The rest of this paper is organized as follows: First, in Section 2, we briefly introduce the relevant auction mechanism. Then the auction model is proposed in Section 3. After that, we give the detailed design of SKMS in the Section 4. In Section 5, we give the conclusion. 


\section{Related work}

In recent years, the literature on the research of keyword advertising has focused on the auction mechanism of keywords from the perspective of search engines. Paid search is gradually becoming a major source of search engine benefits. In 2002, Google launched AdWords products which successfully introduced the generalized second price auction(Generalized Second-Price, GSP) ${ }^{[3]}$ to the keyword advertising auction. The main difference between generalized second price auction and generalized first price auction is the different payment methods. In addition, generalized second-price auction solves the problem of large fluctuations in the bid of advertiser.

Myerson presents a classical optimal auction mechanism for multiple buyers and single item Auction ${ }^{[4]}$. There are few literatures on the mechanism design theory to study the keyword auction ${ }^{[5]}$. Ozluk $\mathrm{O}$ and Y Zhou studied how to allocate the budget so that each keyword bid reasonable ${ }^{[6]}$. Rusmevichientong $\mathrm{P}$ proposed an approximation algorithm to find a set of key words that maximize profit based on historical experience. However, the price of each keyword bidding and advertising remains unchanged, and this situation is not in line with the actual situation $^{[7]}$.

In this paper, we propose that SKMS is mainly concerned with the assignment of a set of synonyms. The search engine defines the different value of each keyword by the different frequency of the keywords which satisfies the computational efficiency, individual rationality and authenticity. Moreover, compared to only one keyword auction, search engines will meet the needs of more advertisers, make full use of Limited advertising and improve social welfare.

\section{System model}

\subsection{Problem description}

Search engine launched the keyword auction in order to better profitability by the advantages of its platform. When netizens need to search for a certain item, they only need to enter keywords in the search engine. Advertisement associated with the keyword will be displayed on web page in a certain order. But search engine can provide a number of synonymous keywords for a variety of products. The value of these synonyms is affected by the frequency of retrieval (the higher the frequency of retrieval, the higher the value of keywords). Such as "computer", "electron brain ", "PC", "electronic computer ", according to the experience, we can know that the frequency of retrieval of "computer" is the highest in these four synonymous keywords. So the value of keyword "computer" is also the highest.

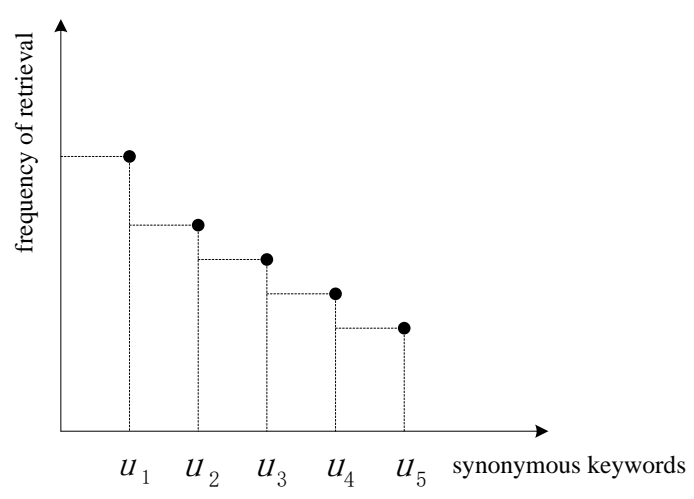

Fig.1. synonymous keywords' frequency of retrieval

As shown in Fig.1, frequency of retrieval of five synonymous keywords $u_{1}, u_{2}, u_{3}, u_{4}, u_{5}$ is $N_{u_{1}}>N_{u_{2}}>$ $N_{u_{3}}>N_{u_{4}}>N_{u_{5}}$. The value of keywords is $V A_{u_{1}}>$ $V A_{u_{2}}>V A_{u_{3}}>V A_{u_{4}}>V A_{u_{5}}$. For advertisers to participate in the auction, they will be assigned to value interval of different synonymous keywords according to their bids. Value interval is defined as $\boldsymbol{\partial}=\left\{\left[V A_{u_{1}},+\infty\right) \rightarrow\right.$ $\left.u_{1},\left[V A_{u_{2}}, V A_{u_{1}}\right) \rightarrow u_{2}, \ldots,\left[V A_{u_{5}}, V A_{u_{4}}\right) \rightarrow u_{5}\right\}$.

\subsection{Model definition}

Search engine provide a set of synonymous keywords $U=\left\{u_{1}, u_{2}, \ldots, u_{n}\right\}$, which if $i<j$, keyword $u_{i}$ appears before keyword $u_{j}$, and $V A_{u_{i}} \geq V A_{u_{j}}$. Each advertiser $v \in V$ has a budget $B_{v}$ and a nonnegative bid $b_{u, v}$ for each keyword $u \in U$. Assume that bid of each advertiser is no more than $B_{v}$. Let $B_{v}(t)$ be the remaining budget of winning advertiser $v$ immediately after the $t-t h$ keyword is assigned to advertiser $v$, and let $b_{u, v}(t)=\min \left(b_{u, v}, B_{v}(t)\right), \quad B_{v}(t)=B_{v}(t-1)-$ $p(t-1), p(t)$ is that advertiser need to pay in this round of auction. For advertisers to participate in the auction, they will be assigned to value interval of different synonymous keywords according to their bids.

For keyword $u_{i}$, search engine provides $k$ advertising sites on each web page. Assume that bid of each advertiser $v$ is either 0 or 1 and $B_{v}=1$. For an advertising site, if advertiser put forward the demand for this advertising site, the bid of advertiser is 1 , otherwise is 0 . If the bid of 
advertiser is less than the value of each keyword, the advertiser will be considered. We can get a 0-1 matrix $\boldsymbol{E}$ according to the above information.

The auctioneer determines the winning advertisers set $V_{\mathrm{w}} \subseteq V$, and the mapping of advertiser and advertising site $\boldsymbol{f}:\left\{i: v_{i} \in V_{w}\right\} \rightarrow\left\{m: l_{m} \in L\right\} . p_{v_{i}}^{(j)}$ and $p_{l_{m}}^{(j)}$ denote the price and payment in $j-t$ h keyword auction, respectively.

Definition 1 (The utility of each advertiser): If advertiser $v_{i}$ wins, the utility of $v_{i}$ is the truthful budget $B_{v_{i}}^{(j)}$ for acquiring the advertising sites, subtracting the payment to search engine in $j-t h$ keyword auction. Otherwise the utility of advertiser $v_{i}$ is zero, i.e.

$$
\boldsymbol{U}_{v_{i}}^{(j)}=\left\{\begin{array}{cc}
B_{v_{i}}^{(j)}-p_{v_{i}}^{(j)}, & \text { if } v_{i} \in V_{w}^{(j)} \\
0, & \text { otherwise. }
\end{array}\right.
$$

Definition 2 (Click Rate): Refers to the number of clicks of an advertising site provided $\boldsymbol{\varphi}$ by search engine percentage of the number of clicks of all advertising site $\boldsymbol{\alpha}$, i.e.

$$
\varepsilon=\varphi / \alpha \times 100 \%
$$

Definition 3 (Recall Rate): The number of advertising sites allocated to advertisers percentage of the number of all advertising sites, i.e.

$$
\theta=\frac{\sum_{j=1}^{n}\left|V_{w}^{(j)}\right|}{n k} \times 100 \%
$$

Definition 4 (Social Welfare): Social welfare is the total budget of all winning advertisers, i.e.

$$
\boldsymbol{S W}=\sum_{j=1}^{n} \sum_{v_{i} \in V_{w}^{(j)}} B_{v_{i}}^{(j)}
$$

Definition 5 (Truthfulness): If advertiser $v_{i}$ gives truthful valuation $b_{u v_{i}}$ and untruthful valuation $b_{u v_{i}}^{\prime}$, acquired utility is $\boldsymbol{U}_{v_{i}}$ and $\boldsymbol{U}_{v_{i}}^{\prime}$ respectively, then a truthful auction mechanism should guarantee $\boldsymbol{U}_{v_{i}} \geq \boldsymbol{U}_{v_{i}}^{\prime}$ regardless of the bid of others, i.e., no advertiser can improve its utility by submitting a bid different from its true valuation.

Definition 6 (Rank of Matrix $)^{[8]}$ : In matrix A, there is $\boldsymbol{r}$-order matrix $\boldsymbol{D}_{\boldsymbol{r}}$ and $\boldsymbol{r}+\mathbf{1}$-order matrix $\boldsymbol{D}_{\boldsymbol{r}+\mathbf{1}}$. When $\left|\boldsymbol{D}_{\boldsymbol{r}}\right| \neq 0$ and $\boldsymbol{D}_{\boldsymbol{r + 1}}=0$ or $\boldsymbol{D}_{\boldsymbol{r}+\mathbf{1}}$ not existing, $\boldsymbol{r}$ will be called rank of matrix $\boldsymbol{A}$, denoted as $\boldsymbol{r}(\boldsymbol{A})$.

The goal in this paper is :

$$
\begin{gathered}
\theta=\max \frac{\sum_{j=1}^{n}\left|V_{w}^{(j)}\right|}{n k} \times 100 \% \\
\boldsymbol{S} \boldsymbol{W}=\max \sum_{j=1}^{n} \sum_{v_{i} \in V_{w}^{(j)}} B_{v_{i}}^{(j)}
\end{gathered}
$$

\section{Synonymous Keywords Auction Mechanism for Advertising Sites (SKMS)}

This part proposes advertising sites allocation algorithm based on 0-1 matrix and payment scheme algorithm mainly for the auction problem of the synonymous keyword meanwhile SKMS is proved of truthfulness.

\subsection{Advertising sites allocation algorithm based on 0-1 matrix}

Algorithm 1 shows the execution flow of the allocation algorithm of Synonymous Keyword Auction Mechanism for Advertising Sites (SKMS).

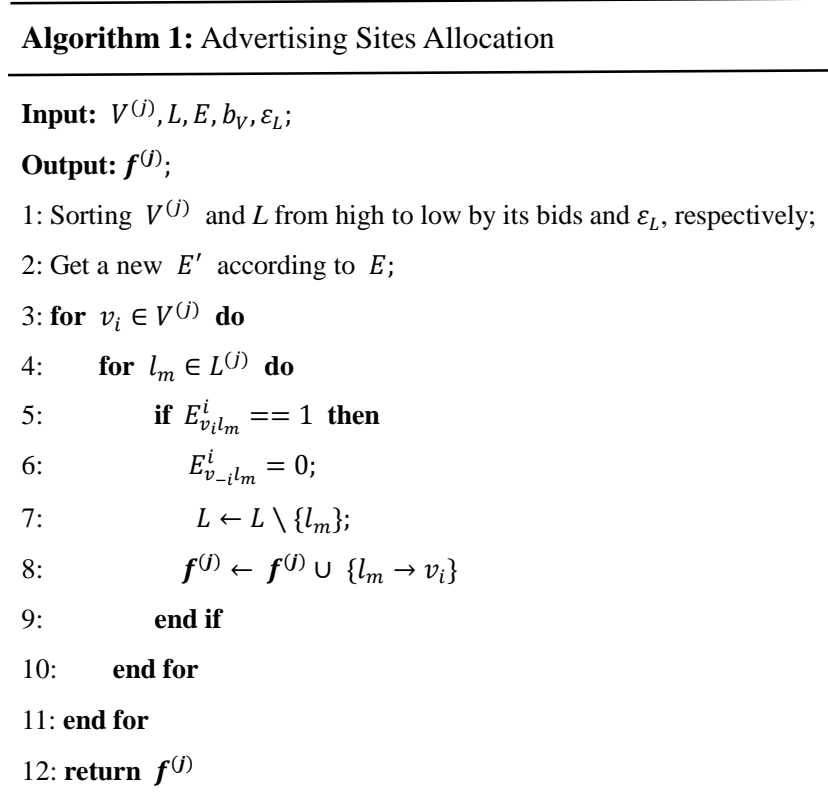

Proposition 1. $r\left(\boldsymbol{f}^{(j)}\right)=\left|L^{(j)}\right| \Leftrightarrow$ Advertising sites are completely allocated for keyword $u_{j}$.

Proof. (1) When advertising sites are completely allocated for keyword $u_{j}, \boldsymbol{f}^{(j)}$ is $\left|L^{(j)}\right|$ order $0-1$ matrix, and each advertising site corresponds to a different advertiser. So $\left|\boldsymbol{f}^{(j)}\right|=1 \neq 0 \Rightarrow r\left(\boldsymbol{f}^{(j)}\right)=\left|L^{(j)}\right|$;

(2) $r\left(\boldsymbol{f}^{(j)}\right)=\left|L^{(j)}\right| \Longrightarrow\left|\boldsymbol{f}^{(j)}\right|=1 \neq 0 \quad$, each advertiser obtains a different advertiser. Because an advertising site cannot be gained simultaneously by multiple advertisers, and an advertiser cannot get multiple advertising sites simultaneously for a keyword, advertising sites are completely allocated for keyword $u_{j}$.

To summarize, $r\left(\boldsymbol{f}^{(j)}\right)=\left|L^{(j)}\right| \Leftrightarrow$ Advertising sites are completely allocated for keyword $u_{j}$.

\subsection{Payment algorithm based on second-price}

Algorithm 2 solves the problem that how to pay for SKMS. According to the mapping of advertisers and advertising sites, payment scheme that based on Second-Price is used. 


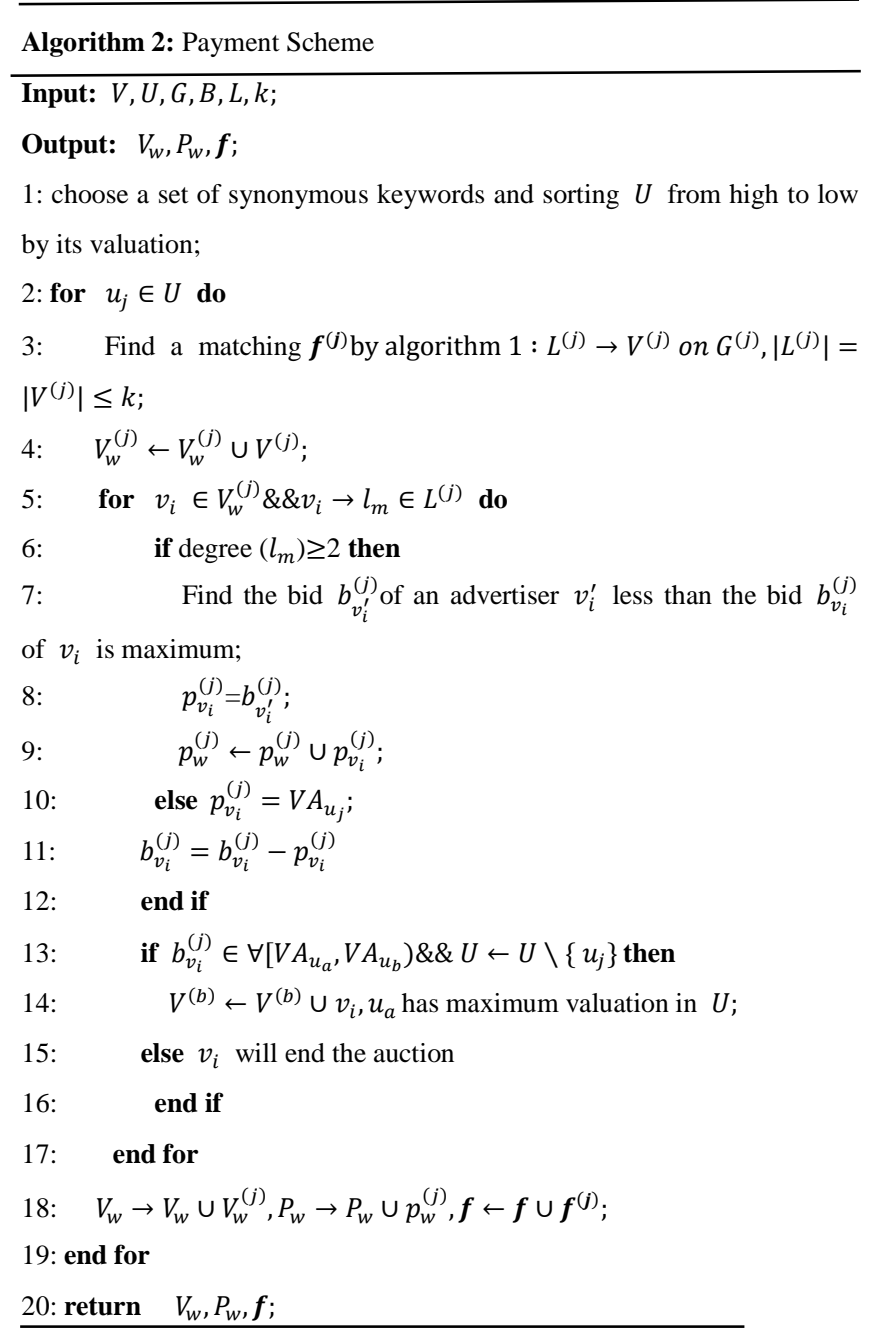

Theorem 1. The utility on the basic of a truthful bid is not less than the utility with an untruthful bid achieved for advertisers, i.e. $\widetilde{U}_{v_{i}}^{(j)} \leq U_{v_{i}}^{(j)}$.

Proof. For each advertiser $v_{i}$ : Assuming advertiser $v_{i}$ wins advertising site $l_{m}$ with truthful bidding. When advertiser $v_{i}$ gives untruthful bidding, two cases should be considered.

(1) Advertiser $v_{i}$ loses: $\widetilde{\boldsymbol{U}}_{v_{i}}^{(j)}=0 \leq \boldsymbol{U}_{v_{i}}^{(j)}$;

(2) Advertiser $v_{i}$ still wins: An advertiser only gets an advertising site for a keyword. Because advertiser $v_{i}$ wins, its bid is still the highest for the advertising site. The payment of advertiser $v_{i}$ is immovable, so $\widetilde{\boldsymbol{U}}_{v_{i}}^{(j)}=b_{v_{i}}^{(j)}-$ $b_{v_{i}^{\prime}}^{(j)}=\boldsymbol{U}_{v_{i}}^{(j)}$ or $\widetilde{\boldsymbol{U}}_{v_{i}}^{(j)}=b_{v_{i}}^{(j)}-V A_{u_{j}}=\boldsymbol{U}_{v_{i}}^{(j)}$.

Therefore, SKMS is truthful.

\section{Conclusion}

In this paper, Synonymous Keyword Auction Mechanism for Advertising Sites (SKMS) is proposed to improve recall rate and increases profit of search engine. In addition, SKMS holds computational efficiency, individual rationality and truthfulness. SKMS brings lower advertising sites of synonymous keywords for advertisers with lower bids. The future work will focus on how to better improve the utilization ratio of advertising sites and revenue of search engine.

\section{Acknowledgment}

Project supported by the National Nature Science Foundation of China (Grant No.61170201, No.61070133, No.61472344); Six talent peaks project in Jiangsu Province (Grant No.2011-DZXX-032). Innovation Foundation for graduate students of Jiangsu Province (Grant No.CXLX12 0916), Jiangsu Science and Technology Project No. BY2015061-06、BY2015061-08, Yangzhou Science and Technology Project No. SXT20140048, SXT20150014, SXT201510013, Natural Science Foundation of the Jiangsu Higher Education Institutions (Grant No.14KJB520041), Innovation Program for graduate students of Jiangsu Province(Grant No.SJZZ16_0261).

\section{References}

(1) Lahaie S, bastien, Pennock D M. Revenue analysis of a family of ranking rules for keyword auctions[C]. ACM Conference on Electronic Commerce. DBLP, 2007:50-56.

(2) Elyakime B, Laffont J J, Loisel P, et al. First-price sealed-bid auction with secret reservation price [J]. Annales Déconomie Et De Statistique, 1994, 34.

(3) Omote K, Miyaji A. A Second-Price Sealed-Bid Auction with Verifiable Discriminant of $\mathrm{p}$, 0-th Root[C]. Financial Cryptography, International Conference, FC 2002, Southampton, Bermuda, March 11-14, 2002, Revised Papers. 2002: page.

(4) Myerson R B. Optimal Auction Design[J]. Mathematics of Operations Research, 1981(1):59—73.

(5) Abrams Z., Mendelevitch O., Tomlin J.: Optimal delivery of sponsored search advertisements subject to budget constraints. In: EC 2007 (2007).

(6) Rusmevichientong P, David P W. An Adaptive Algorithm for Selecting Profitable Keywords for Search-based Advertising Services[c]. Proceedings of the $7^{\text {th }}$ ACM Conference on Electronic Commerce. New York, USA: ACM Press, 2006:260-269.

(7) Cary M, Das A, Edelman B. Greedy Bidding Strategies for Keyword Auctions[c]. Proceedings of the $8^{\text {th }}$ ACM Conference on Electronic Commerce. New York. USA: ACM Press.2007:262-271.

(8) Cragg J G, Donald S G. Inferring the rank of a matrix $[\mathrm{J}]$. Journal of Econometrics, 1997, 76(12):223-250. 